\title{
Research Paper \\ Effect of Lower Extremity Fatigue on Knee Joint Kinematics During Landing Maneuvers in Adult Soccer Players
}

\author{
Zahra Khazaee $^{1}$ (i), ${ }^{*}$ Mehdi Gheitasi ${ }^{2}$ (), Amir Hosein Barati ${ }^{2}$
}

1. Department of Sport Injuries and Corrective Exercises, Faculty of Health and Sport Sciences, Shahid Beheshti University, Tehran, Iran. 2. Department of Health \& Rehabilitation in Sport, Faculty of Health and Sport Sciences, Shahid Beheshti University, Tehran, Iran.

\begin{tabular}{|c|c|}
\hline $\begin{array}{l}\text { Use your device to scan } \\
\text { and read the article online }\end{array}$ & \\
\hline 口ifing & $\begin{array}{l}\text { Citation Khazaee Z, Gheitasi M, Barati AH. [Effect of Lower Extremity Fatigue on Knee Joint Kinematics During Landing Maneu- } \\
\text { vers in Adult Soccer Players (Persian)]. Scientific Journal of Rehabilitation Medicine. 2021; 10(3):562-573. https://doi.org/10.32598/ } \\
\text { sjrm.10.3.15 }\end{array}$ \\
\hline aptequs & dol'https://doi.org/10.32598/sjrm.10.3.15 \\
\hline
\end{tabular}

Received: 21 Jan 2020

Accepted: 18 Aug 2020 Available Online: $23 \mathrm{Jul} 2021$

Keywords:

Kinematics, Fatigue, Landing, Soccer player

\section{A B STR ACT}

Background and Aims Since fatigue in different regions of the lower extremities can effectively alter the movement pattern of this part of the body and cause joint-related kinematic changes that increase the risk of injury and mental fatigue, which may be a factor in reducing productivity and injury. Therefore, the study of kinematic changes during fatigue can have helpful results. This study aimed to investigate the effect of lower extremity fatigue on knee joint kinematics during landing in adult soccer players. Methods Ten adult male soccer players (Mean \pm SD = age: $20.7 \pm 1.05$ years; Height: $178.9 \pm 4.17 \mathrm{~cm}$; Weight: $71.55 \pm 8.04 \mathrm{~kg}$ ) participated in this study. Subjects performed a pre-test, which included jumping and landing on a 40-cm box, and recording cameras of the valgus motion analyzer, flexion, and knee rotation. The Kingtools section of the plugin software attached to the Cortex software was used for segmentation and kinematic information. The lower extremity fatigue protocol consisted of 10 repetitions of single-leg squats up to 90 degrees of knee flexion, 20 vertical jumps with a single leg, and 1 repetition of step -going up and down a $31 \mathrm{~cm}$ step. The Borg scale was used to measure fatigue. Before and after the fatigue protocol, a single-leg hop was used to determine the level of fatigue. After the fatigue protocol, a post-test was performed. Data were analyzed using the Shapiro-Wilk test for normality of the data, and paired t-test was used to compare mean in pre-test and post-test independent variables.

Results The paired t-test results for comparison of kinematic data showed that contact flexion had a significant difference from pre-test to post-test and other kinematic variables had no significant changes from pre-test to post-test. Statistical significance was considered at $\mathrm{P} \leq 0.05$.

Conclusion According to the findings of this study, it can be concluded that lower extremity fatigue in the present study partly caused kinematic changes in predicting ACL injury. Kinematic changes have included a decrease in Contact flexion, which is one of the predictors of ACL injury during landing.

\section{* Corresponding Author: \\ Mehdi Gheitasi, PhD.}

Address: Department of Health \& Rehabilitation in Sport, Faculty of Health and Sport Sciences, Shahid Beheshti University, Tehran, Iran

Tel: +98 (912) 3547613

E-Mail: m_gheitasi@sbu.ac.ir 


\section{Extended Abstract}

\section{Introduction}

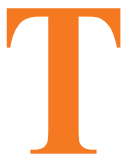

One of the most common injuries in sports is Anterior Cruciate Ligament (ACL) rupture. Research shows that noncontact mechanisms cause about $70 \%$ of ACL injuries. Also, the video images show that the probability of ACL rupture of athletes during landing and cutting movements is higher than forwarding movements and, of course, other movement maneuvers. Recognizing risk factors is the first step in controlling and preventing sports injuries. Fatigue refers to a temporary decrease in the ability to produce strength or power during sports activities.

Fatigue is one of the factors that can lead to ACL injury. Fatigue is thought to reduce muscle strength, and consequently, negative changes in the kinematics and kinetics of the hip, knee, and ankle joints, and may increase the pressure on the ACL during dangerous movements such as cutting or landing lead to injury Increase. Fatigue is one of the factors that can play an essential role in the performance of footballers during their activity to perform their best performance and effort and even cause serious injury to the athlete. According to research conducted in the final minutes of a football match, especially in the last 15 minutes of each half, injury prevalence is very high, and many researchers believe that most ACL injuries can be prevented by changing the biomechanics of movement in the athlete.

Reducing the flexion angle of the knee joint along with increasing quadriceps muscle activity simultaneously puts more shear force into the ACL, which increases the likelihood of rupture. This study aimed to evaluate the effect of lower extremity fatigue on knee joint kinematics during landing in adult footballers.

\section{Methods}

The method of the present study, considering the application of the intervention variable, is to select the subjects purposefully based on the criteria of entry and exit of the pre-experimental type. The statistical population of the present study was adult male football players working in clubs in Tehran province in the age range of 19 to 23 years. The samples of this study were purposefully selected from 10 members of the mentioned community and were included in the study based on the inclusion criteria. After being selected, the participants went to the laboratory of Shahid Beheshti University and entered the experimental stages after registering their details. Before performing the test, each subject performed a warm-up for 5 minutes.
To perform the landing test, the subject stood on a box 40 $\mathrm{cm}$ high and landed on the ground with a pair of feet at the examiner's command. Each test records the correct landing task three times. Kinematic data collection of the present study was performed using a 3D motion analyzer (7 cameras) motion analysis made in the United States. Data were collected at a sampling rate of 240 .

Helen Hayes marking method was used to select landmarks for pasting infrared markers. Marking was performed before the test, and after the pre-test, the subjects performed the lower extremity fatigue protocol. The measured kinematic variables included valgus, flexion, and knee rotation at initial contact and peak flexion. The lower extremity fatigue protocol consisted of 10 repetitions of one-legged squat up to 90 degrees of knee flexion, 2 vertical jumps with a maximum of one leg, and 20 repetitions of step up and down a 31-centimeter step. This set of exercises was a set of protocols. The subject was asked to express fatigue level between each set using a scale of 0 to 10 Borg. When a person announced the number 10 after a few sets, he was given a Single Leg fore distance test to ensure fatigue.

Before and after the fatigue protocol, the subjects performed a single-leg for distance test to determine the amount of distance, which was a criterion for measuring fatigue. In this study, the Kintools section of the plugin software, attached to the Cortex software, was used for segmentation and kinematic information. After collecting data, the Shapiro-Wilk test (for statistical samples less than 50 people have a higher statistical power) to examine the natural distribution of data, paired t-test was used to compare the mean in pre-test and post-test independent variables. A statistically significant difference was also determined at the level of $\mathrm{P} \leq 0.05$.

\section{Results}

The results of Shapiro-Wilk test showed a normal distribution of data $(\mathrm{P} \geq 0.05)$. The paired t-test to compare the data showed that the initial contact flexion had a significant difference in pre-test and post-test, and other kinematic variables did not have significant changes in pre-test and post-test stages.

\section{Discussion and Conclusion}

The present study results showed that initial contact flexion decreased significantly after applying fatigue protocol, and other kinematic variables did not show significant changes. These results indicate that lower extremity fatigue caused a change in knee flexion angle during initial contact and a significant reduction in knee flexion at the initial contact on the left leg after applying fatigue pro- 
tocol in the muscles of the lower extremities. Changes in body position on the sagittal plane affect the biomechanics of the trunk and lower extremity and the activation of the lower extremity muscles. Landing with a smooth posture increases the reaction force of the ground, the maximum torque of the knee openers, and the activation of the quadriceps muscles while reducing the flexion angle of the knee and reducing the torque of the thigh extensors. Increases the load on the anterior cruciate ligament. Fatigue is a neuromuscular factor that has been linked to knee injuries, according to previous research. Concludingly, the cause of fatigue through kinematic changes in the lower limb joints, especially in the knee joint in the form of reduction (knee flexion) and increase (knee valgus) knee movements in the frontal and horizontal planes can provide an injury mechanism, especially for ACL.

\section{Ethical Considerations}

\section{Compliance with ethical guidelines}

The ethical principles observed in the article, such as the informed consent of the participants, the confidentiality of information, the permission of the participants to cancel their participation in the research. Ethical approval was obtained from the Research Ethics Committee of the Research Ethics Committee of Sports Science Research Institute (Code: IR.SSRC.REC.1398.123).

\section{Funding}

This study was extracted from the MSc. thesis of the first author at the Department of Health and rehabilitation in sports of Faculty of Sport Science \& Health, Shahid Beheshti University.

Authors' contributions

Authors contributed equally in preparing this article.

\section{Conflict of interest}

The authors declared no conflict of interest. 
مقاله بزووهشى

\title{
اثر خستًّ اندام تحتانى بر كينماتيك مفصل زانو طى مانور فرود در فوتباليستهاي مرد بزرَّال
}

\author{
زهرا خزائى' هـ، "مهدى قيطاسى' هـ امير حسين براتى' هـ \\ 1. كروه آسيبشئاسي ورزشي و حركات اصلاحي، دانشكده علوم ورزشي و تثندرستي، دانشكاه شهيد بهشتي، تهران، ايران.

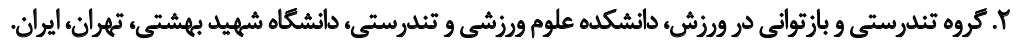

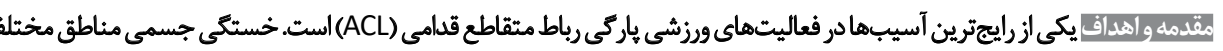

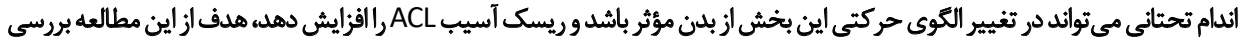

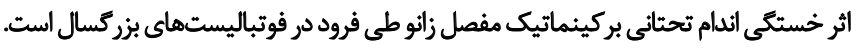

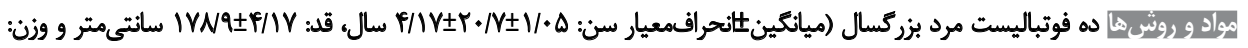

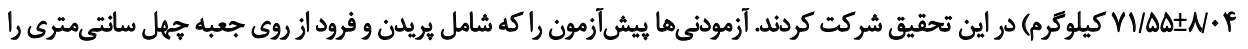

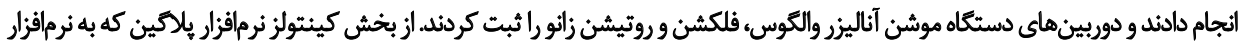

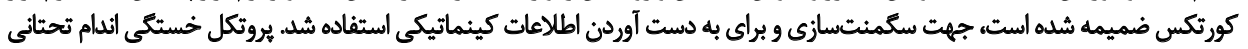

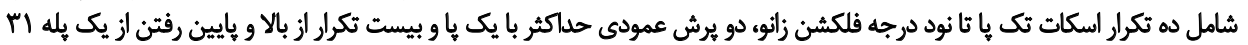

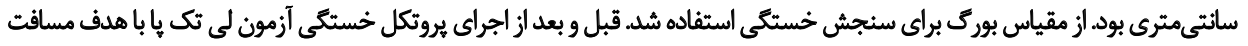

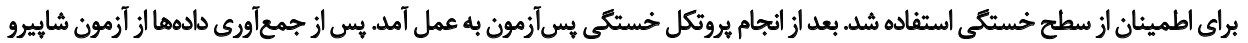

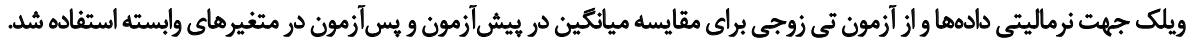

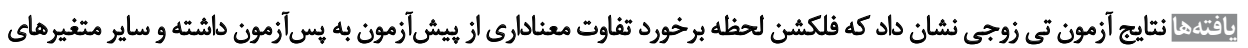

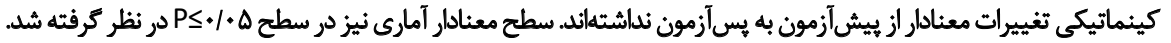

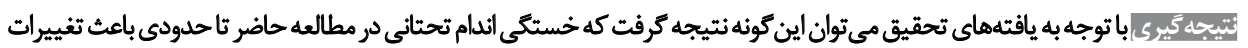

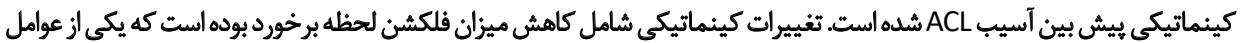
بيشبين آسيب ACL هنكام فرود است.

تاريخ دريافت: ال• بهمن

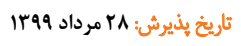

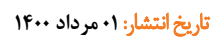

آسيبهاى ACL ناشى از سازوكارهاى غيربرخوردى است [بائ.

dale

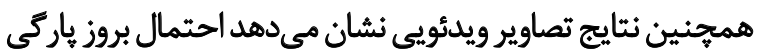

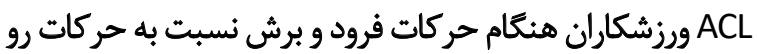

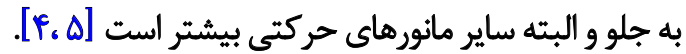

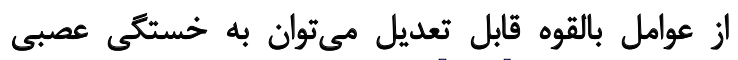

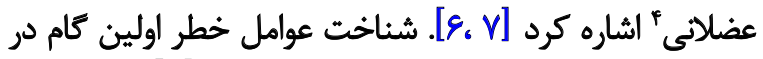

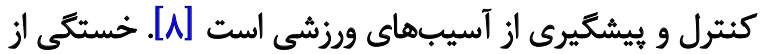

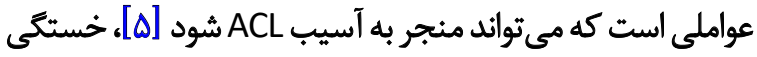

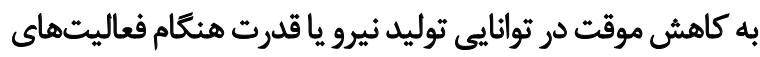


كه خم كردن بيشتر در وضعيث فرود، ناشى از افزايش فلكشن إز رائن

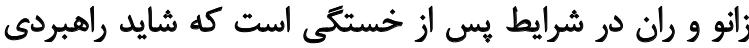

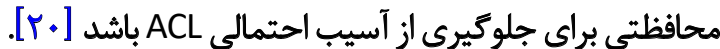

بنجامين و همكاران، تغييرات كينماتيك اندام تحثانى در ياثزئه

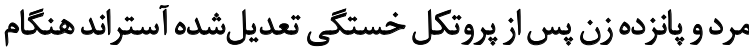

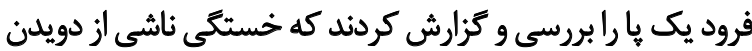

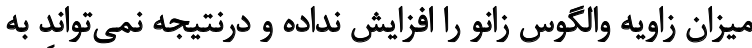

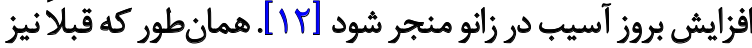

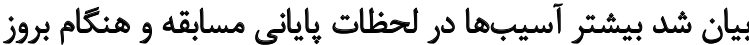

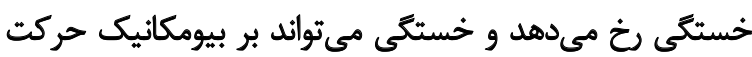

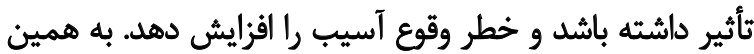

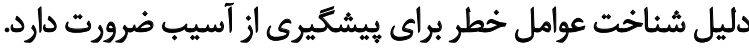

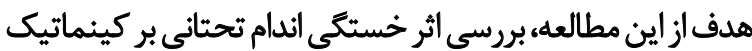
مفصل زانو هنكام فرود در فوتباليستهاي بزر بركال است.

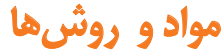

روش تحقيق حاضر با توجه ببه اعمال متغير مداخلداى يروتكل

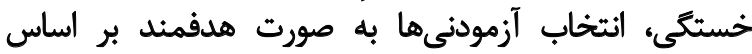

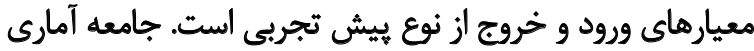

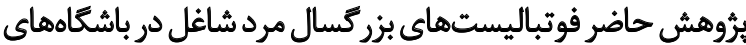

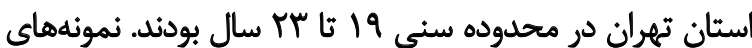

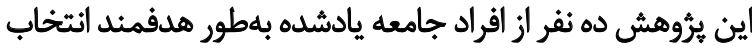

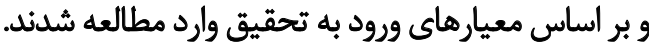

اين معيارها شامل [Yl] سابقه فعاليت در رشته ورزشى فوتبال

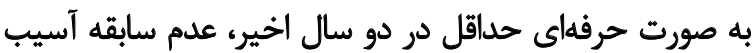

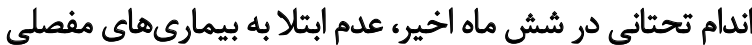

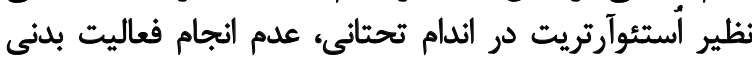

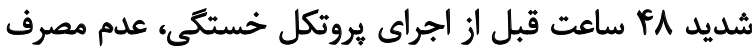

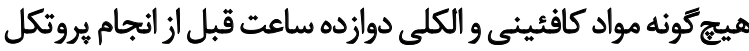

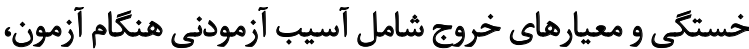

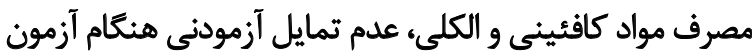

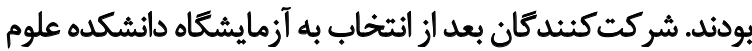

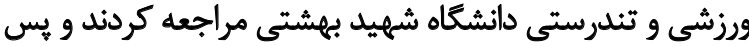

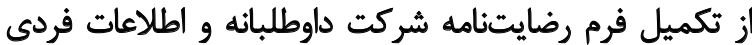

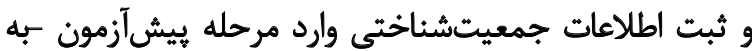

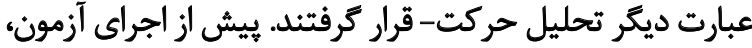

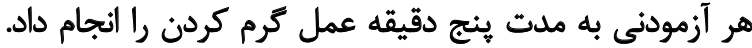

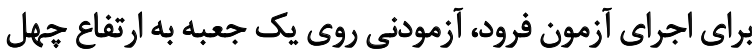

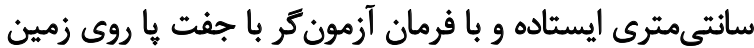

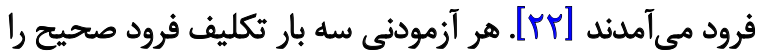

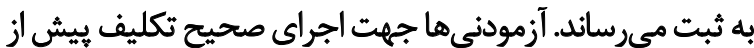

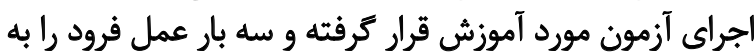
صورت تمرينى اجرا مي مردند.
ورزشى اشاره دارد. سازوكارهاى ايجاد خستّى را مىتوان به انواع

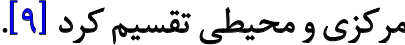

بخش بزركى از خستكى عضلاتى ناشى از فرايندهاي داخلى

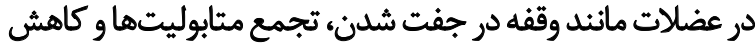

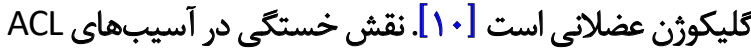

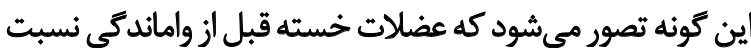

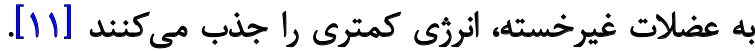

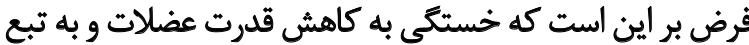

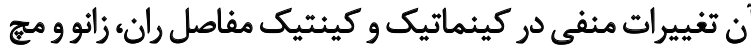

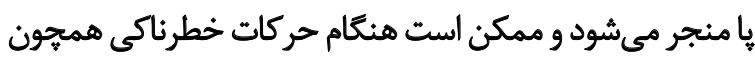

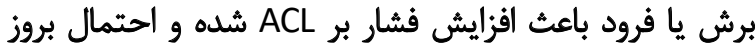

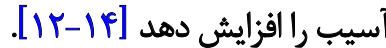

بسيارى از محققان اعتقاد دارند كه بيشتر آسيبهاى ACL با بياري

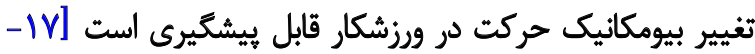

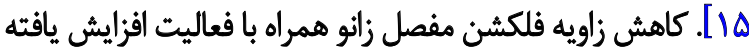

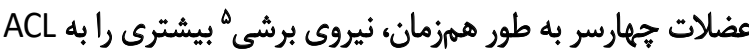

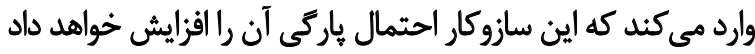

[10]

نتايج مطالعات بويشين نشان ميدهند كه خستخى يكى از

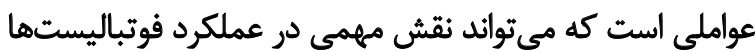

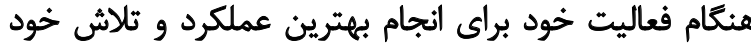

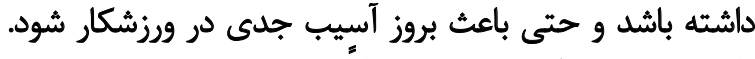

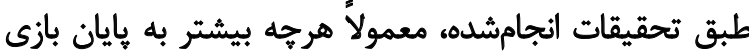

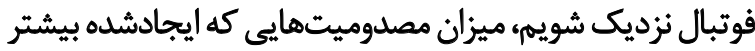

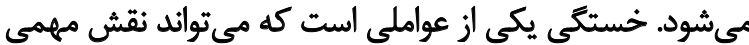

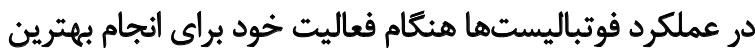

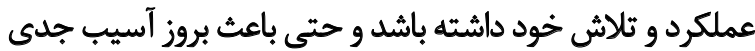

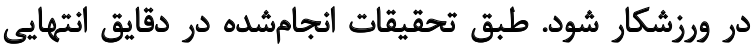

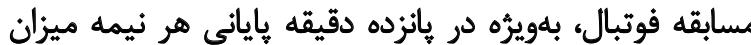

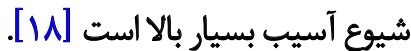
بريم و همكاران در مطالعهاى روى ورزشكاران كودك دختر

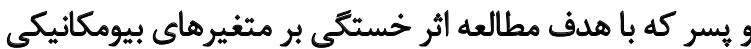

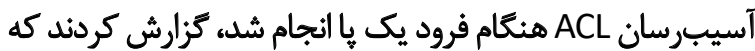

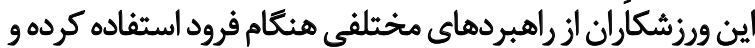

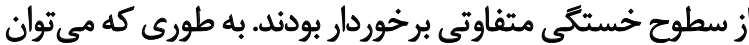

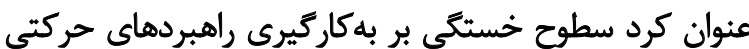

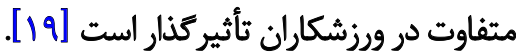

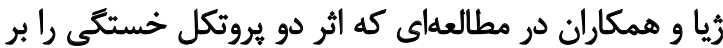

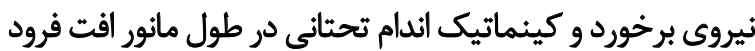

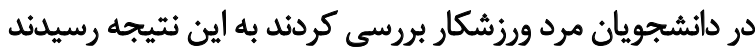

5. Shearing Force 


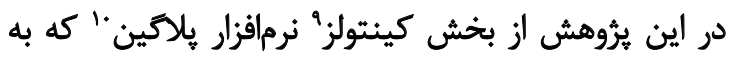

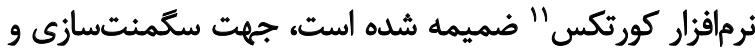

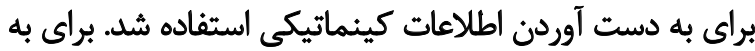

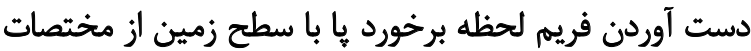

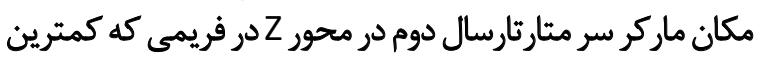

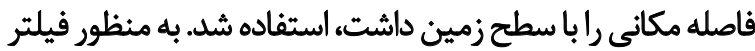

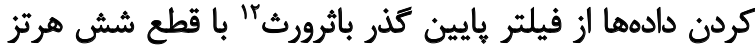

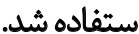

$$
\text { روش هاي آمارى }
$$

يس از جمعآورى دادهها از آزمون شابيرو ويلك (براى

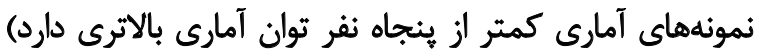

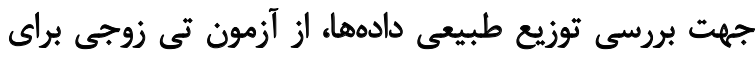

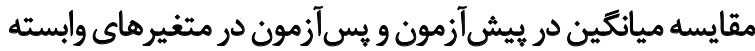

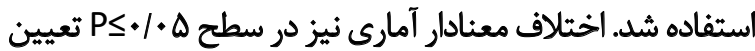

شد.

L.)

در جدول شماره | اطلاعات جمعيتشناختى شركت كنيندكان

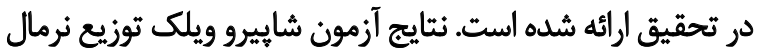

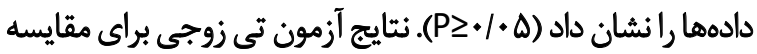

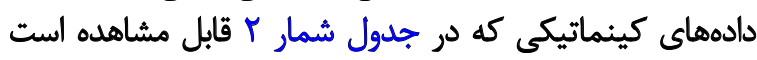

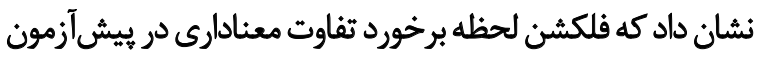

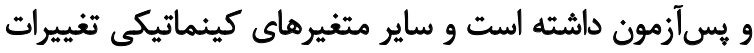

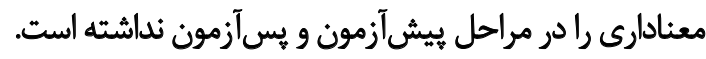

ث잉

هدف از اين مطالعه، بررسى اثر خستگى اندام تحتانى بر

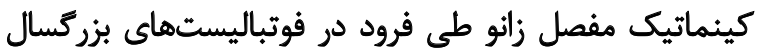

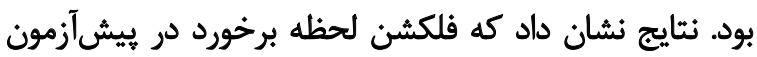

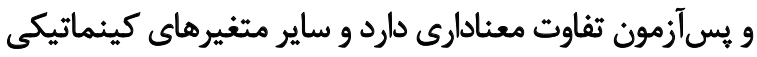
تغييرات معنادارى رانشان نمى دهند.

اين نتايج بيانكر اين مسئله است كه خستخى اندام تحتانى

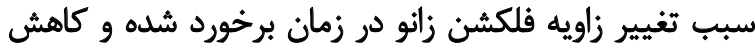

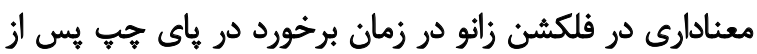

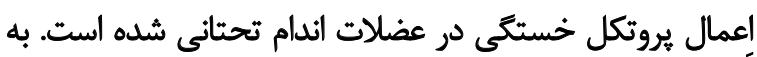

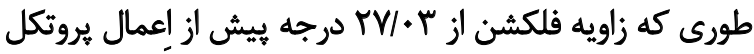

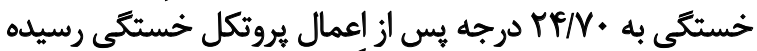

9. Kin Tools

10. Plugin

11. Cortex

12. Butterworth
جمعآورى اطلاعات كينماتيكى تحقيق حاضر با استفاده از

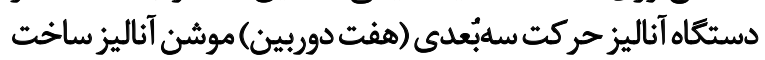

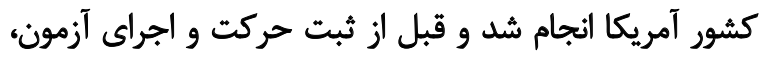

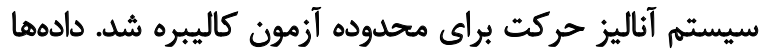

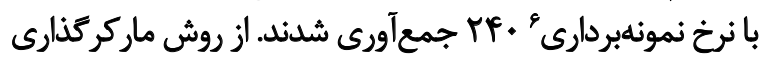

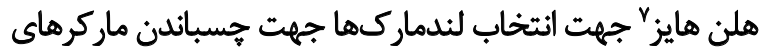

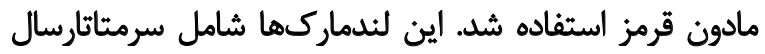

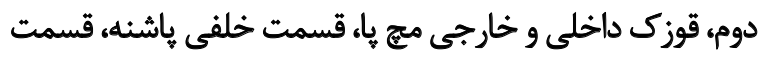

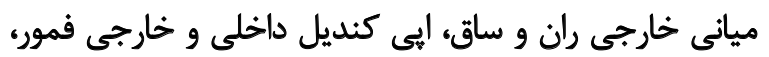

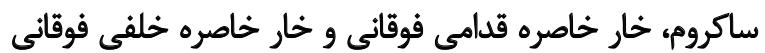

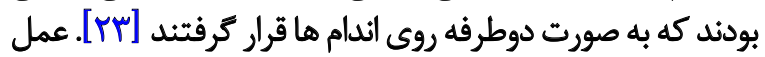

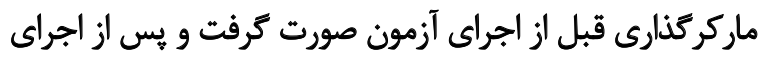

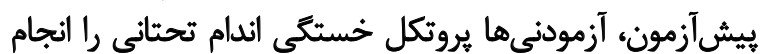

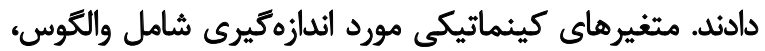

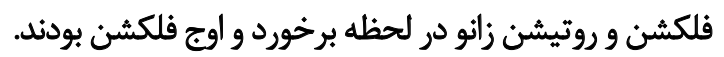

$$
\text { يروتكل خُشتكي اندام تحمثاثي. }
$$

يروتكل خستكى اندام تحتانى شامل ده تكرار اسكات تك يا يا تا

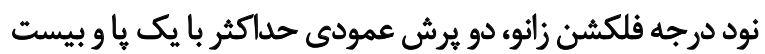

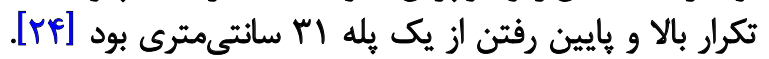

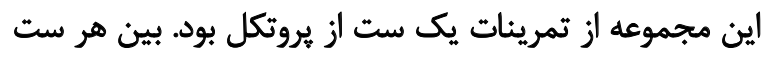

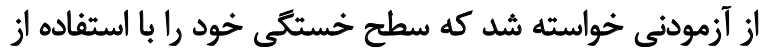

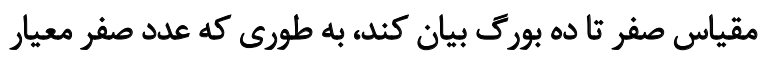

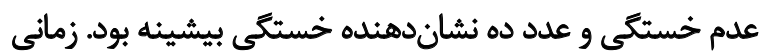

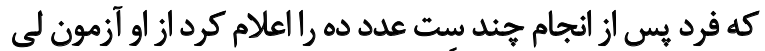

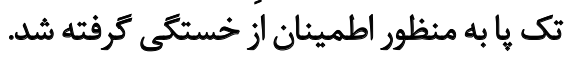

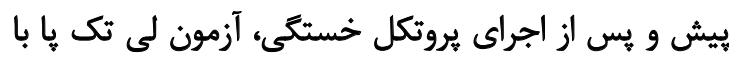

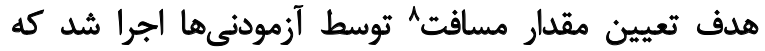

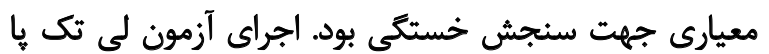

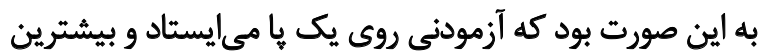

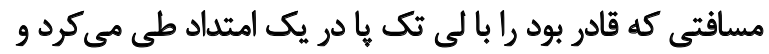

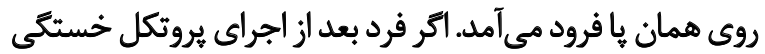

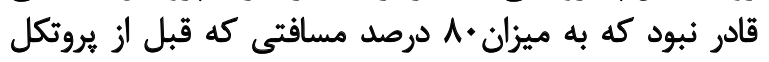

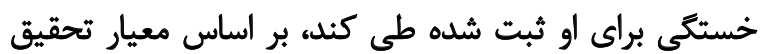

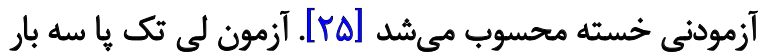

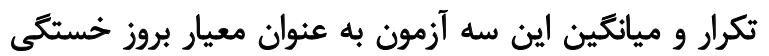

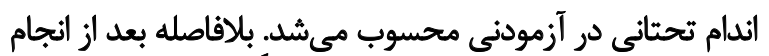

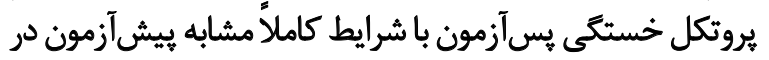

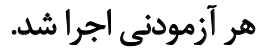

8. Single Hop for Distance Test 
جدول ا. ويرّكى هاى جمعيتششناختى آزمودنى هاي مورد مطالعه

ميانكين |انحراف استاندارد

كروه خستكي اندام تحثانى

هتغيرها

IVNQ $4 / / Y$

قد (سايتى متر)

$V / \Delta \Delta \pm N \cdot r$

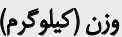

$r \cdot N \pm V+\Delta$

(سال) (س)

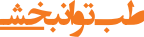

دهد [YN] كورتس و همكاران، تغييرات كينماتيك روى هجده

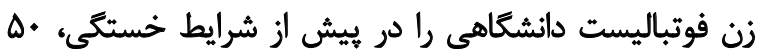

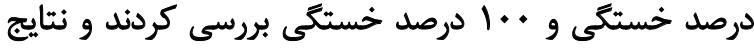

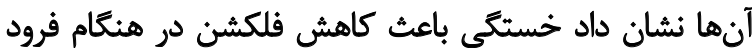

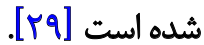

از نتايج مخالف با فرضيه اين تحقيق، مطالعه ابركل كه شرايط

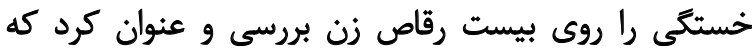

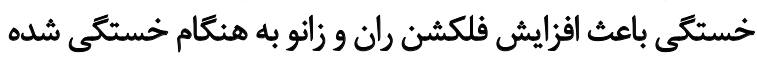

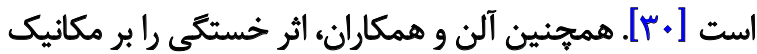

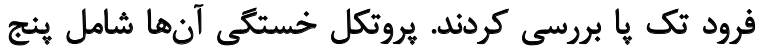

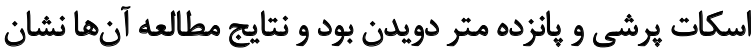

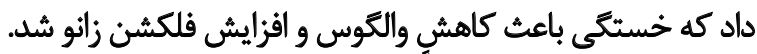

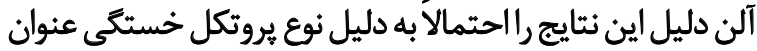

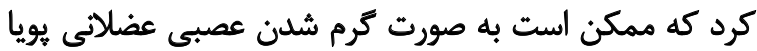

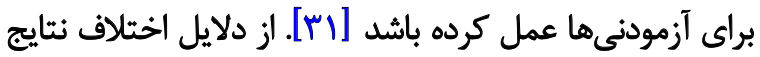

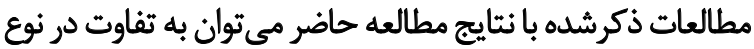

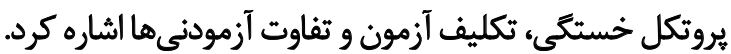
اكرجه در مطالعه حاضر تغييرات در والكوس يس از إعمال

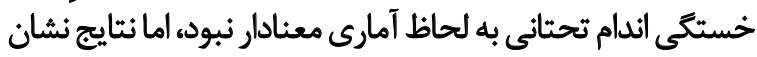

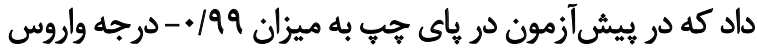

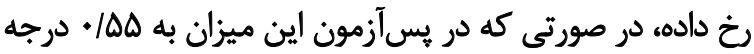

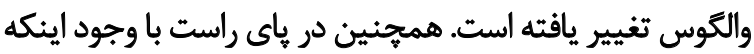

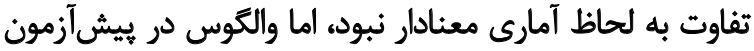

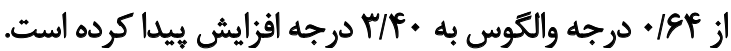

مي توان افزايش حركت مشاهدهشه والكوس در صفحه فرونتال

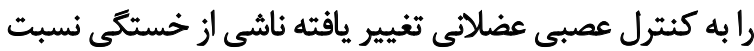

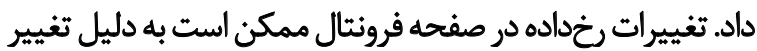

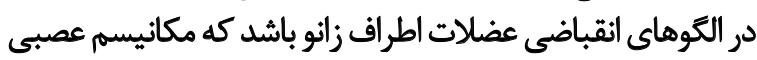

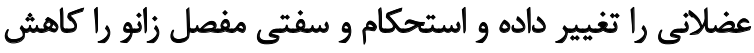

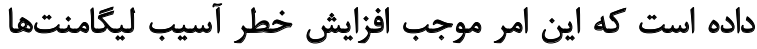

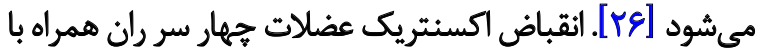

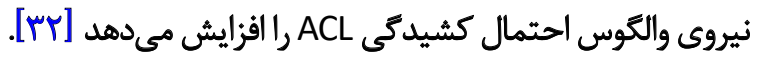
از مطالعات همسو با نتايج تحقيق حاضر مىتوان به تحقيق
است. از اين نتايج مى توان اين كونه استنباط كرد كه كنترل زانو

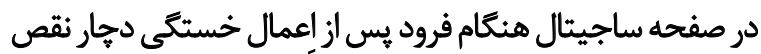
و تغيير شده، به جز والكوس لحظه برخورد فروده افزرايش يافته است.

مورد ديكرى كه در تحقيق حاضر مورد توجه است، معنادار

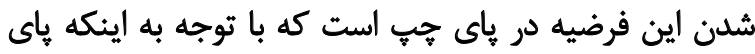

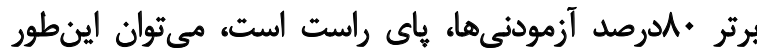

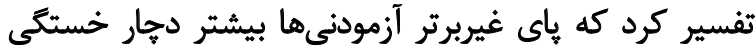

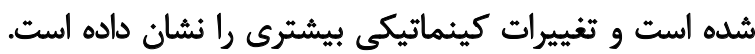

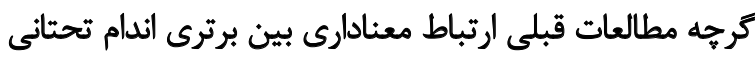

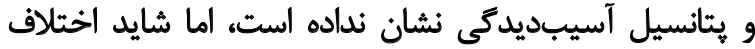

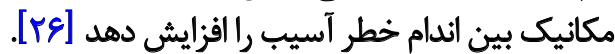

ساير فاكتورهاى كينماتيكى يس از إعمال يروتكل خستيكى

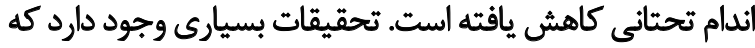

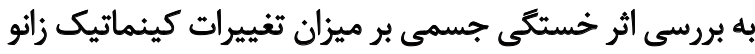

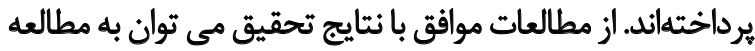

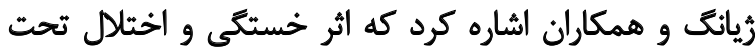

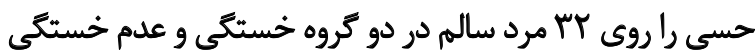

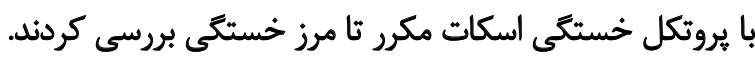

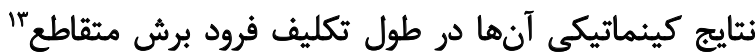

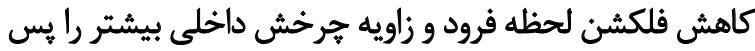

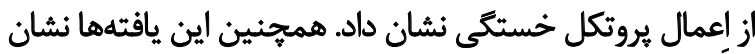
داد كه برش متقاطع خطرناكتر از فرود در وضعيت خستكي إنى

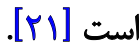

تغيير موقعيت بدن در صفحه ساجيتال، بيومكانيك تثنه و اندام

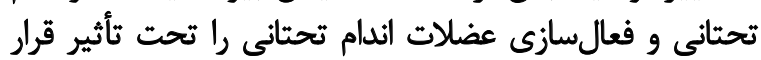

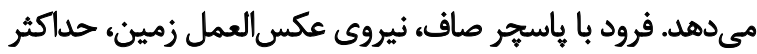

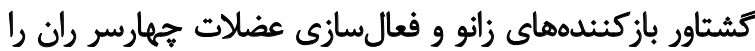

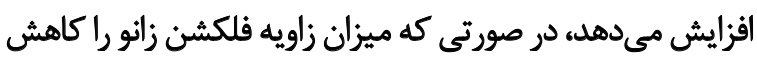

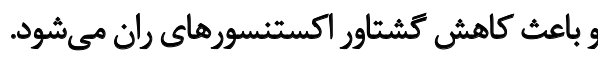
كاهش زاويه فلكشن زانو هنكام فرود شايد ميزان بار وارده

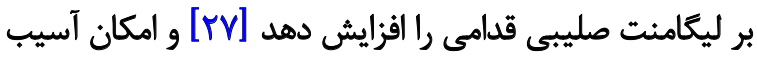

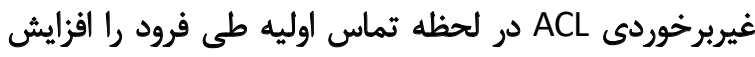




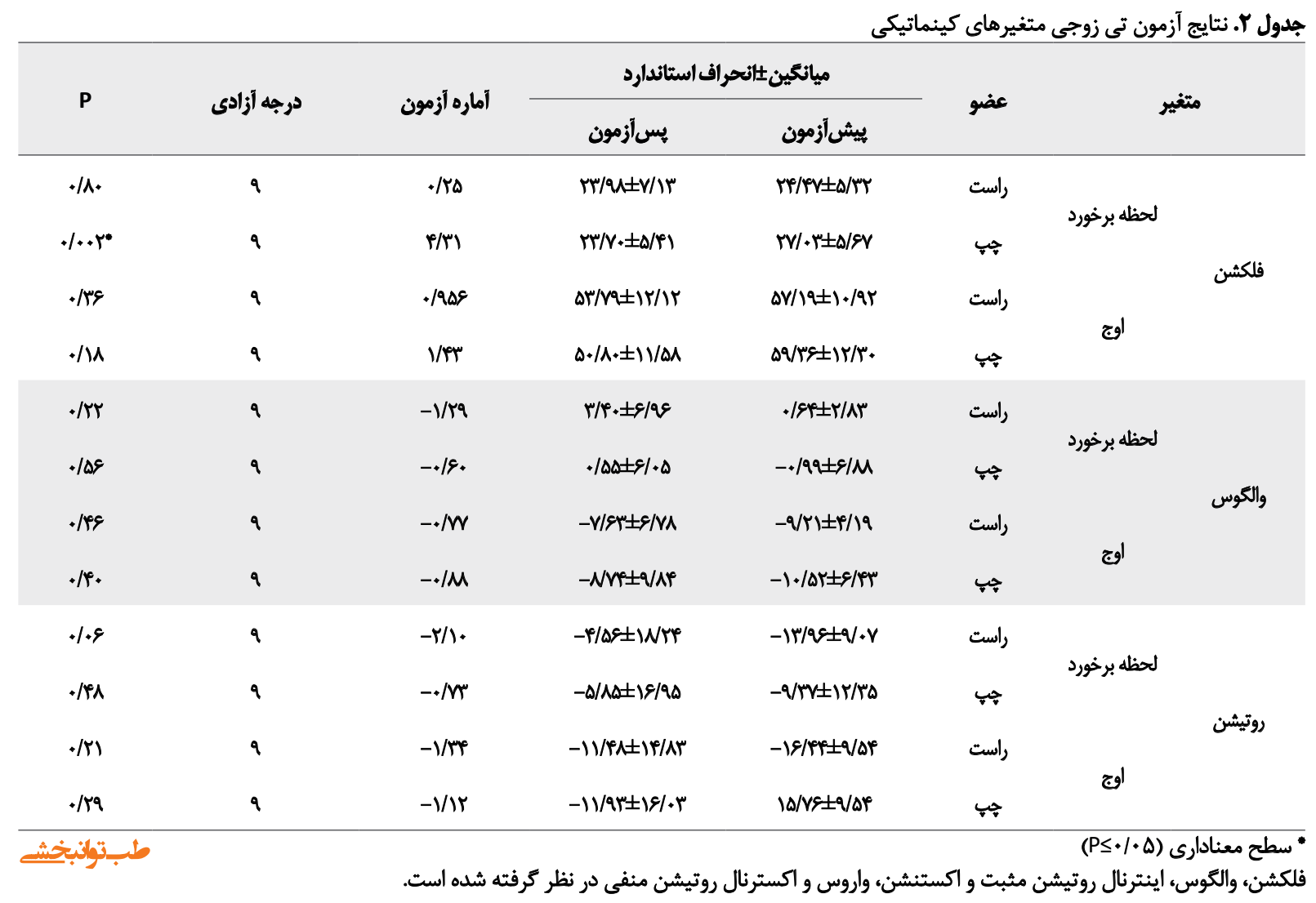

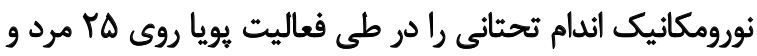

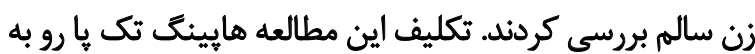

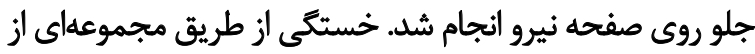

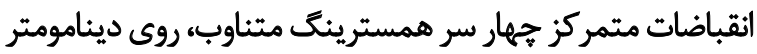

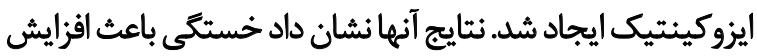

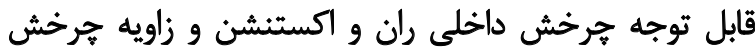

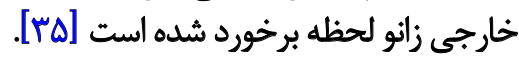

\section{نتيجه يَيرى نمبايى}

خستَّى يك عامل عصبى عضلانى است كه با توجه به نتايج

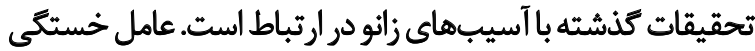

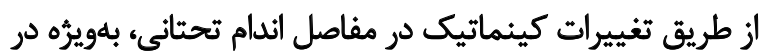

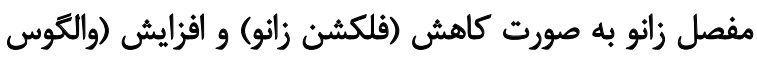

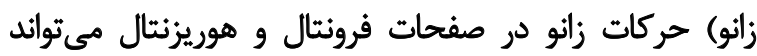

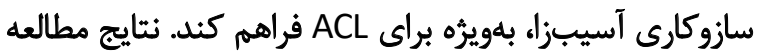

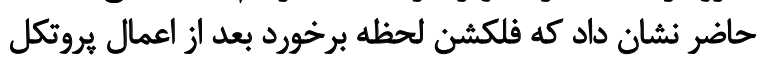

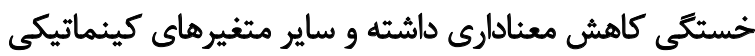
تغييرات معنادارى نشان ندادهاند.
كرنوزى و همكاران اشاره كرد كه اثر تفاوت جنسيتى را در

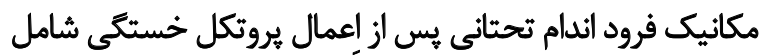

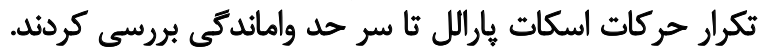

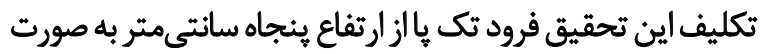

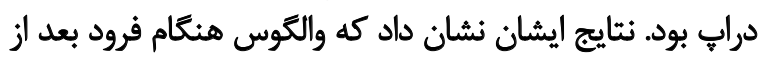

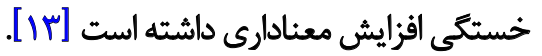

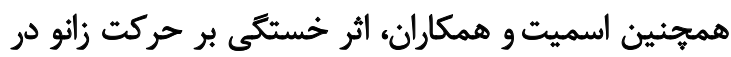

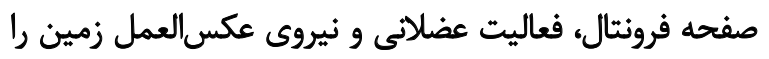

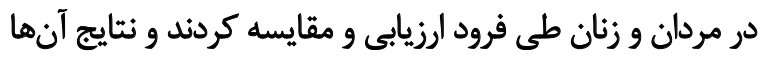

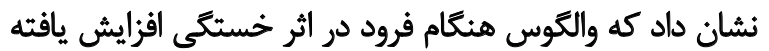

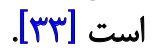

از ديكر نتايج مطالعه حاضر، كاهش جرخش خار خارجى و و تمايل

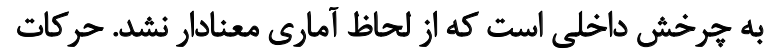

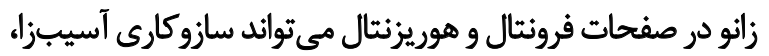

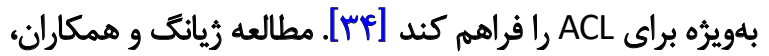

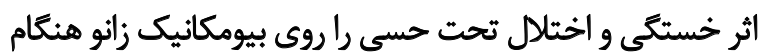

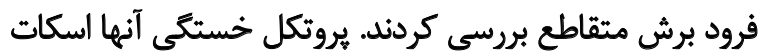

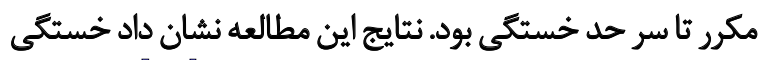

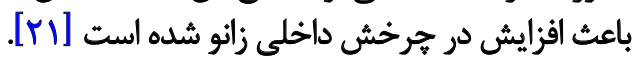
از مطالعات ناهمسو با نتايج اين مطالعه، مطالعهاي است

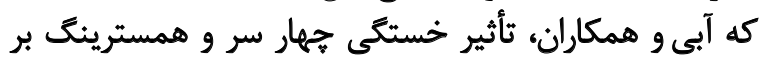




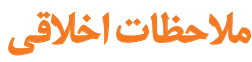

$$
\text { يميووى أز أصول اخلاق يثوهش }
$$

در اجراى يُروهش ملاحظات اخلاقي مطابق با دستورالعمل

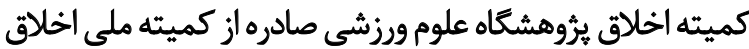

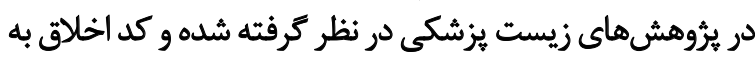
شماره IR.SSRC.REC.1398.123 دريافت شده است كرئه

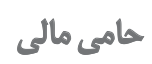

اين مقاله بركرفته از بايانناملى نويسنده اول در تروها

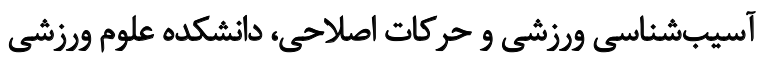
و تندرستى، دانشكاه شهيد بهشتى، تهران است.

$$
\text { مشاركت ثويسئدكان }
$$

تمام نويسندكان در آمادهازي اين مقاله مشاركت يكسان

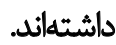

$$
\text { تعارض مثافع }
$$

بنابر اظهار نويسندكان اين مقاله تعارض منافع ندارد. 


\section{References}

[1] Majewski M, Susanne $H$, Klaus S. Epidemiology of athletic knee injuries: A 10-year study. The Knee. 2006; 13(3):184-8. [DOI:10.1016/j.knee.2006.01.005] [PMID]

[2] Hewett TE, Ford KR, Hoogenboom BJ, Myer GD. Understanding and preventing acl injuries: Current biomechanical and epidemiologic considerations-update 2010. North American Journal of Sports Physical Therapy: NAJSPT. 2010; 5(4):234-51. [PMCID]

[3] Jamison ST, Pan X, Chaudhari AM. Knee moments during runto-cut maneuvers are associated with lateral trunk positioning. Journal of Biomechanics. 2012; 45(11):1881-5.[DOI:10.1016/j. jbiomech.2012.05.031] [PMID]

[4] Kirkendall DT, Garrett WE. The anterior cruciate ligament enigma: Injury mechanisms and prevention. Clinical Orthopaedics and Related Research. 2000; 372:64-8. [DOI:10.1097/00003086-200003000-00008] [PMID]

[5] Boden BP, Dean GS, Feagin JA, Garrett WE. Mechanisms of anterior cruciate ligament injury. Orthopedics. 2000; 23(6):5738. [DOI:10.3928/0147-7447-20000601-15] [PMID]

[6] Barber-Westin SD, Noyes FR. Effect of fatigue protocols on lower limb neuromuscular function and implications for anterior cruciate ligament injury prevention training: A systematic review. The American Journal of Sports Medicine. 2017; 45(14):3388-96. [DOI:10.1177/0363546517693846] [PMID]

[7] Benjaminse A, Webster KE, Kimp A, Meijer M, Gokeler A. Revised approach to the role of fatigue in anterior cruciate ligament injury prevention: A systematic review with metaanalyses. Sports Medicine. 2019; 49(4):565-86. [DOI:10.1007/ s40279-019-01052-6] [PMID] [PMCID]

[8] Bahr R, Engebretsen L. Sports injury prevention. United States: Wiley Online Library; 2009. https://onlinelibrary.wiley.com/ doi/book/10.1002/9781444303612

[9] Enoka RM, Duchateau J. Muscle fatigue: What, why and how it influences muscle function. The Journal of Physiology. 2008; 586(1):11-23. [DOI:10.1113/jphysiol.2007.139477] [PMID] [PMCID]

[10] Deschenes MR, Maresh CM, Kraemer WJ. The neuromuscular junction: Structure, function, and its role in the excitation of muscle. Journal of Strength and Conditioning Research. 1994; 8(2):103-9.[DOI:10.1519/00124278-199405000-00008]

[11] Mair SD, Seaber AV, Glisson RR, Garrett JR WE. The role of fatigue in susceptibility to acute muscle strain injury. The American Journal of Sports Medicine. 1996; 24(2):137-43. [DOI:10.1 177/036354659602400203] [PMID]

[12] Benjaminse A, Habu A, Sell TC, Abt JP, Fu FH, Myers JB, et al. Fatigue alters lower extremity kinematics during a single-leg stop-jump task. Knee Surgery, Sports Traumatology, Arthroscopy. 2008; 16(4):400-7. [DOI:10.1007/s00167-007-0432-7] [PMID]

[13] Kernozek TW, Torry MR, Iwasaki M. Gender differences in lower extremity landing mechanics caused by neuromuscular fatigue. The American Journal of Sports Medicine. 2008; 36(3):554-65. [DOI:10.1177/0363546507308934] [PMID]
[14] Wojtys EM, Beaulieu ML, Ashton-Miller JA. New perspectives on ACL injury: On the role of repetitive sub-maximal knee loading in causing ACL fatigue failure. Journal of Orthopaedic Research. 2016; 34(12):2059-68. [DOI:10.1002/jor.23441] [PMID] [PMCID]

[15] Bell DR, Smith MD, Pennuto AP, Stiffler MR, Olson ME. Jump-landing mechanics after anterior cruciate ligament reconstruction: A landing error scoring system study. Journal of Athletic Training. 2014; 49(4):435-41. [DOI:10.4085/10626050-49.3.21] [PMID] [PMCID]

[16] DiStefano LJ, Padua DA, DiStefano MJ, Marshall SW. Influence of age, sex, technique, and exercise program on movement patterns after an anterior cruciate ligament injury prevention program in youth soccer players. The American Journal of Sports Medicine. 2009; 37(3):495-505. [DOI:10.1177/0363546508327542] [PMID]

[17] Hewett TE, Di Stasi SL, Myer GD. Current concepts for injury prevention in athletes after anterior cruciate ligament reconstruction. The American Journal of Sports Medicine. 2013; 41(1):216-24. [DOI:10.1177/0363546512459638] [PMID] [PMCID]

[18] Yoon YS, Chai M, Shin DW. Football injuries at Asian tournaments. The American Journal of Sports Medicine. 2004 32(S1):36S-42. [DOI:10.1177/0095399703258781] [PMID]

[19] Briem K, Jónsdóttir KV, Árnason Á, Sveinsson P. Effects of sex and fatigue on biomechanical measures during the drop-jump task in children. Orthopaedic Journal of Sports Medicine. 2017; 5(1):2325967116679640.[DOI:10.1177/2325967116679640] [PMID] [PMCID]

[20] Xia R, Zhang X, Wang X, Sun X, Fu W. Effects of two fatigue protocols on impact forces and lower extremity kinematics during drop landings: Implications for noncontact anterior cruciate ligament injury. Journal of Healthcare Engineering. 2017; 2017:5690519. [DOI:10.1155/2017/5690519] [PMID] [PMCID]

[21] Qu X, Jiang J, Hu X. Effects of subsensory noise and fatigue on knee landing and cross-over cutting biomechanics in male athletes. Journal of Applied Biomechanics. 2018; 34(3):20510. [DOI:10.1123/jab.2017-0180] [PMID]

[22] Frank BS, Gilsdorf CM, Goerger BM, Prentice WE, Padua DA Neuromuscular fatigue alters postural control and sagittal plane hip biomechanics in active females with anterior cruciate ligament reconstruction. Sports Health. 2014; 6(4):301-8. [DOI:10.1177/1941738114530950] [PMID] [PMCID]

[23] Northeast L, Gautrey CN, Bottoms L, Hughes G, Mitchell AC, Greenhalgh A. Full gait cycle analysis of lower limb and trunk kinematics and muscle activations during walking in participants with and without ankle instability. Gait \& Posture. 2018 64:114-8. [DOI:10.1016/j.gaitpost.2018.06.001] [PMID]

[24] Lessi GC, Dos Santos AF, Batista LF, de Oliveira GC, Serrão FV. Effects of fatigue on lower limb, pelvis and trunk kinematics and muscle activation: Gender differences. Journal of Electromyography and Kinesiology. 2017; 32:9-14. [DOI:10.1016/j. jelekin.2016.11.001] [PMID]

[25] Orishimo KF, Kremenic IJ. Effect of fatigue on single-leg hop landing biomechanics. Journal of Applied Biomechanics. 2006; 22(4):245-54. [DOI:10.1123/jab.22.4.245] [PMID] 
[26] Negrete RJ, Schick EA, Cooper JP. Lower-limb dominance as a possible etiologic factor in noncontact anterior cruciate ligament tears. Journal of Strength and Conditioning Research. 2007; 21(1):270-3. [DOI:10.1519/00124278-20070200000048] [PMID]

[27] Cortes N, Greska E, Kollock R, Ambegaonkar J, Onate JA. Changes in lower extremity biomechanics due to a short-term fatigue protocol. Journal of Athletic Training. 2013; 48(3):30613. [DOI:10.4085/1062-6050-48.2.03] [PMID] [PMCID]

[28] Lucci S, Cortes N, Van Lunen B, Ringleb S, Onate J. Knee and hip sagittal and transverse plane changes after two fatigue protocols. Journal of Science and Medicine in Sport. 2011; 14(5):453-9. [DOI:10.1016/j.jsams.2011.05.001] [PMID] [PMCID]

[29] Cortes N, Greska E, Ambegaonkar JP, Kollock RO, Caswell SV, Onate JA. Knee kinematics is altered post-fatigue while performing a crossover task. Knee Surgery, Sports Traumatology, Arthroscopy. 2014; 22(9):2202-8. [DOI:10.1007/s00167-0132673-y] [PMID] [PMCID]

[30] Abergel R. The effects of fatigue on lower extremity landing mechanics in dancers. United States: California State University; 2019. https://books.google.com/books/about/The_Effects_ of_Fatigue_on_Lower_Extremit.html?id=fM1bzgEACAAJ

[31] Allen R, Chick M, Grossman M, Phillips S. The effects of fatigue on single leg landing mechanics. 2019. https://digitalcommons.ithaca.edu/cgi/viewcontent. cgi?article $=1664 \&$ context $=$ whalen

[32] Chappell JD, Creighton RA, Giuliani C, Yu B, Garrett WE. Kinematics and electromyography of landing preparation in vertical stop-jump: risks for noncontact anterior cruciate ligament injury. The American Journal of Sports Medicine. 2007; 35(2):235-41. [DOI:10.1177/0363546506294077] [PMID]

[33] Smith MP, Sizer PS, James CR. Effects of fatigue on frontal plane knee motion, muscle activity, and ground reaction forces in men and women during landing. Journal of Sports Science \& Medicine. 2009; 8(3):419-27. [PMCID]

[34] Chavez A. The effect of fatigue on ACL injury risk in the athletic population [MSc. Thesis]. Texas: Texas State University; 2011. https://digital.library.txstate.edu/handle/10877/4326

[35] Thomas AC, McLean SG, Palmieri-Smith RM. Quadriceps and hamstrings fatigue alters hip and knee mechanics. Journal of Applied Biomechanics. 2010; 26(2):159-70. [DOI:10.1123/ jab.26.2.159] [PMID] 
This Page Intentionally Left Blank 